\title{
Article
}

\section{Quantifying the origin and distribution of intracluster Light in a Fornax-Like Cluster}

Harris, Kathryn A., Debattista, Victor P, Governato, Fabio, Thompson, Benjamin B., Clarke, Adam, Quinn, Thomas, Willman, Beth, Benson, Andrew, Farrah, Duncan, Peng, Eric W., Elliott, Rachel and Petty, Sara

Available at http://clok.uclan.ac.uk/18981/

Harris, Kathryn A., Debattista, Victor P ORCID: 0000-0001-7902-0116, Governato, Fabio, Thompson, Benjamin B., Clarke, Adam ORCID: 0000-00023299-8034, Quinn, Thomas, Willman, Beth, Benson, Andrew, Farrah, Duncan et al (2017) Quantifying the origin and distribution of intracluster Light in a Fornax-Like Cluster. Monthly Notices of the Royal Astronomical Society, 467 (4). pp. 4501-4513. ISSN 0035-8711

It is advisable to refer to the publisher's version if you intend to cite from the work. http://dx.doi.org/10.1093/mnras/stx401

For more information about UCLan's research in this area go to http://www.uclan.ac.uk/researchgroups/ and search for <name of research Group>.

For information about Research generally at UCLan please go to http://www.uclan.ac.uk/research/

All outputs in CLoK are protected by Intellectual Property Rights law, including Copyright law. Copyright, IPR and Moral Rights for the works on this site are retained by the individual authors and/or other copyright owners. Terms and conditions for use of this material are defined in the policies page. 


\title{
Quantifying the origin and distribution of intracluster Light in a Fornax-Like Cluster
}

\author{
Kathryn A. Harris, ${ }^{1 \star}$ Victor P. Debattista, ${ }^{2 \star}$ Fabio Governato, ${ }^{3}$ \\ Benjamin B. Thompson, ${ }^{2}$ Adam J. Clarke, ${ }^{2}$ Thomas Quinn, ${ }^{3}$ Beth Willman, ${ }^{4}$ \\ Andrew Benson, ${ }^{5}$ Duncan Farrah, ${ }^{1}$ Eric W. Peng, ${ }^{6,7}$ Rachel Elliott ${ }^{1}$ and Sara Petty ${ }^{1}$ \\ ${ }^{1}$ Department of Physics, Virginia Tech, Blacksburg, VA 24061, USA \\ ${ }^{2}$ Jeremiah Horrocks Institute, University of Central Lancashire, Preston PR1 2HE, UK \\ ${ }^{3}$ Astronomy Department, University of Washington, WA 98195, USA \\ ${ }^{4}$ Haverford College, Haverford, PA 19041, USA \\ ${ }^{5}$ The Observatories of the Carnegie Institution for Science, 813 Santa Barbara St, Pasadena, CA 91101, USA \\ ${ }^{6}$ Department of Astronomy, Peking University, Beijing 100871, China \\ ${ }^{7}$ Kavli Institute for Astronomy and Astrophysics, Peking University, Beijing 100871, China
}

Accepted 2017 February 14. Received 2017 February 12; in original form 2015 February 5

\begin{abstract}
Using a cosmological $N$-body simulation, we investigate the origin and distribution of stars in the intracluster light (ICL) of a Fornax-like cluster. In a dark-matter-only simulation, we identify a halo that, at $z=0$, has $M_{200} \simeq 4.1 \times 10^{13} \mathrm{M}_{\odot}$ and $r_{200}=700 \mathrm{kpc}$, and replace infalling subhalos with models that include spheroid and disc components. As they fall into the cluster, the stars in some of these galaxies are stripped from their hosts, and form the ICL. We consider the separate contributions to the ICL from stars that originate in the haloes and the discs of the galaxies. We find that disc ICL stars are more centrally concentrated than halo ICL stars. The majority of the disc ICL stars are associated with one initially disc-dominated galaxy that falls to the centre of the cluster and is heavily disrupted, producing part of the $\mathrm{cD}$ galaxy. At radial distances greater than $200 \mathrm{kpc}$, well beyond the stellar envelope of the $\mathrm{cD}$ galaxy, stars formerly from the stellar haloes of galaxies dominate the ICL. Therefore at large distances, the ICL population is dominated by older stars.
\end{abstract}

Key words: methods: numerical - galaxies: clusters: general-galaxies: clusters: intracluster medium - cosmology: theory.

\section{INTRODUCTION}

Intracluster light (ICL), discovered by Zwicky (1951), is light from stars within a galaxy cluster that are not bound to a galaxy. This light, which has been observed in clusters up to $z=0.8$ (Guennou et al.2012), constitutes between 2 per cent and 50 per cent of a cluster's total light (Arnaboldi 2004; Feldmeier et al. 2004a; Lin \& Mohr 2004; Zibetti 2008; McGee \& Balogh 2010; Toledo et al. 2011). The origin of these stars is thought to be the cluster galaxies themselves; the stars being stripped from the galaxies as they orbit within the cluster (e.g. White 1978; Merritt 1984; Moore et al. 1996; Calcáneo-Roldán et al. 2000; Arnaboldi 2004; Zibetti \& White 2005; Conroy \& Gunn 2007). Indeed the network of tidal features observed in the core of the Virgo cluster supports the view that stripped stars are a major contributor to the ICL (Mihos et al. 2005). Nonetheless, an ICL component formed in situ out of gas has also been suggested. For instance, the simulations of Puchwein et al.

^E-mail: kateharris142@gmail.com (KAH); VPDebattista@uclan.ac.uk (VPD)
(2010), which include active galactic nucleus feedback, find that as much as 30 percent of the ICL may have formed in situ. However, there may be an upper limit to how much ICL light in situ star formation can produce. While gas being stripped from infalling galaxies is sometimes seen to be forming stars (e.g. Sun et al. 2010; Zhang et al. 2011), stellar population synthesis of stacked spectra of the ICL gives an upper limit to a younger ICL population of $\sim 1$ per cent (Melnick et al. 2012).

The ICL is an important diagnostic of processes such as the enrichment of the intracluster medium (Lin \& Mohr 2004; Cora 2006; Sivanandam et al. 2009) that affects cooling flows (Peterson et al. 2003), the evolution of baryonic substructures (Arnaboldi \& Gerhard 2010) and the baryon budget in clusters and groups (Gonzalez, Zubludoff \& Zaritsky 2005; Gonzalez, Zaritsky \& Zubludoff 2007). The ICL has also been used to trace the structure of dark matter (DM): Jee (2010) used the ICL and weak lensing to trace a ring-like DM structure near the centre of the cluster CL0024+17. Giallongo et al. (2014) compared observations of the ICL to a DM model and concluded that it can be used to probe the DM distribution. 
The ICL fraction depends on galaxy richness, increasing from $<2$ per cent for loose groups (e.g. Feldmeier et al. 2003, 2004a) to $>20$ per cent for rich clusters (e.g. Feldmeier et al. 2002; Lin \& Mohr 2004). This effect is also seen in some simulations (Rudick, Mihos \& McBride 2011). Other simulations, however, have found a fairly constant ICL fraction with cluster richness (e.g. Puchwein et al. 2010). The semi-analytic model of Purcell, Bullock \& Zentner (2007) finds an ICL fraction rising rapidly to $\sim 20$ per cent in haloes with mass up to $10^{13} \mathrm{M}_{\odot}$, but that this rise becomes much less dramatic at higher masses, reaching only $\sim 30$ per cent at $10^{15} \mathrm{M}_{\odot}$. The semi-analytic model of Contini et al. (2014) instead produces no halo mass dependence.

Although the ICL can make up to half of the cluster light, it is much more diffuse than the light from the galaxies (e.g. Feldmeier et al. 2002; Guennou et al. 2012), making it difficult to detect. Instead, the ICL is often studied using discrete, bright tracers such as planetary nebulae (e.g. Arnaboldi et al. 1996; Arnaboldi 2005; Aguerri et al. 2005; Castro-Rodriguéz et al. 2009; Mihos et al. 2009), red giants (e.g. Ferguson, Tanvir \& von Hippel 1998; Durrell et al. 2002; Palladino et al. 2012), globular clusters (e.g. Williams et al. 2007; Peng et al. 2011; Durrell et al. 2014), novae (e.g. Neill, Shara \& Oegerle 2005; Shara 2006) and supernovae (e.g. Smith 1981; Gal-Yam et al. 2003; McGee \& Balogh 2010; Sand et al. 2011). These methods, however, require assumptions about the light from the underlying stellar population in order to convert between the observed number of tracers and the total stellar mass in the ICL.

Numerical simulations have been used to study the ICL. These include the creation and evolution of the ICL (Rudick, Mihos \& McBride 2006; Puchwein et al. 2010; Cooper et al. 2015), the kinematics of unbound stars (e.g. Murante et al. 2004; Willman et al. 2004; Dolag, Murante \& Borgani 2010), the ICL fraction (e.g. Murante et al. 2004; Willman et al. 2004; Rudick et al. 2011; Martel, Barai \& Brito 2012), ICL substructures (e.g. CalcáneoRoldán et al. 2000; Murante et al. 2004; Rudick et al. 2011) and the radial distribution of the ICL (e.g. Napolitano et al. 2003; Rudick et al. 2009; Guennou et al. 2012). A common feature of most of these simulations is that they focus on high-mass clusters, generally $>10^{14} \mathrm{M}_{\odot}$. However, high-mass clusters are atypical, with most galaxies residing in lower mass associations, such as loose groups or clusters. These lower mass clusters have several potentially key density and kinematic differences with their high-mass cousins (e.g. Forman \& Jones 1990). For example, the Fornax cluster, though less massive than the Virgo cluster $\left(\sim 10^{13}-10^{14} \mathrm{M}_{\odot}\right.$, as opposed to Virgo's $\sim 10^{15} \mathrm{M}_{\odot}$; Ikebe et al. 1992; Drinkwater, Gregg \& Colless 2001; Fouqué et al. 2001; Nasonova, de Freitas Pacheco \& Karachentsev 2011; Lee, Kim \& Rey 2015), has approximately three times the density of galaxies within its core compared to Virgo (Davies et al. 2013), and a lower velocity dispersion of the giant galaxies $\left(\sim 370 \mathrm{~km} \mathrm{~s}^{-1}\right.$ compared to $\sim 1000 \mathrm{~km} \mathrm{~s}^{-1}$; Ftaclas, Struble \& Fanelli 1984; Binggeli, Popescu \& Tammann 1993; Drinkwater et al. 2000, 2001; Kim et al. 2014). Fornax also appears to be more dynamically evolved than Virgo (Churazov et al. 2008). Because of these differences, coupled with the way the ICL may be formed (via tidal interactions and stripping from galaxies), we may expect different ICL compositions and distributions in lower mass clusters.

This paper investigates the distribution and origin of ICL stars in a cluster comparable to the Fornax cluster using an $\mathrm{N}$-body simulation. This simulated cluster is of a lower mass than has been studied in previous ICL papers, with a total stellar mass of $\sim 10^{11} \mathrm{M}_{\odot}$ and DM mass of $\sim 10^{13} \mathrm{M}_{\odot}$. We investigate whether ICL stars originate primarily from the discs or the haloes of progenitor galaxies. Separating the ICL into stars that originated from the disc (younger) and halo (older) of galaxies allows us to further investigate the idea that the ICL stars may be older than the stellar populations in surviving galaxies (e.g. Murante et al. 2004). Section 2 describes the simulation used and the formation of part of the cD galaxy. In Section 3, methods used to image and identify the ICL are described. The results are presented in Section 4 including the radial distribution of the ICL and its components, the ICL luminosity and the stellar age of the ICL. Section 5 discusses these results and presents our conclusions. In the appendix, we present the individual galaxies and their orbits within the cluster.

\section{$2 N$-BODY METHODS}

We explore the ICL via a cosmological simulation using PKDGRAV (Stadel 2001) of 18 galaxies within a Fornax cluster-like environment. We start with a DM-only simulation, evolved in a WMAP (Spergel et al. 2003) $\Lambda$ cold dark matter cosmology with $\Omega_{0}=0.3$, $\Omega_{\Lambda}=0.7$ and $H_{0}=70 \mathrm{~km} \mathrm{~s}^{-1} \mathrm{Mpc}^{-1}$. The initial simulation has a low resolution of $36^{3}$ particles in a cube of size $70 h^{-1} \mathrm{Mpc}$. We then identify a cluster with a virial radius $r_{200} \simeq 700 \mathrm{kpc}$ and virial mass $M_{200} \simeq 4.1 \times 10^{13} \mathrm{M}_{\odot}$. These properties make the cluster similar to the Fornax cluster. We then use the zoom-in technique (Katz \& White 1993) to resimulate at high resolution the formation of the Fornax-sized halo. The resolution of the base simulation is refined in four nested steps by factors of $2^{3}, 2^{3}, 4^{3}$ and $3^{3}$ centred on the cluster. At the highest resolution, this gives a total of $15492788 \mathrm{DM}$ particles. The particle masses range from $7.9 \times 10^{6}$ to $8.7 \times 10^{11} \mathrm{M}_{\odot}$, with corresponding particle softenings varying from 0.13 to $6.2 \mathrm{kpc}$.

At the end of this process, we identified all haloes in the mass range of $8.6 \times 10^{10} \leq M_{200} \leq 5.2 \times 10^{12} \mathrm{M}_{\odot}$ entering the cluster between redshift $z=1.65$ and 0.13 (before $z=1.65$ the cluster is too chaotic to allow easy replacement). Before they enter, we replace 18 of these haloes with full galaxy models. We exclude only two haloes from this replacement because they are strongly interacting as they enter the cluster. Because strong interactions eject stars to large radii (Hilz et al. 2012; Hilz, Naab \& Ostriker 2013) where they are more easily stripped in a cluster environment, excluding these haloes will lead to an underestimate of the ICL. Material that fell into the cluster earlier than $z=1.6$ would probably have ended up in the central cD galaxy (Diemand, Madau \& Moore 2005). Thus the stellar content of the central object in our simulation is underestimated. Our replacement mass cut-off for $M_{200}>8.6 \times$ $10^{10} \mathrm{M}_{\odot}$ roughly corresponds to a stellar mass cut of $\sim 10^{9} \mathrm{M}_{\odot}$, equivalent to $<0.1 L_{*}$ at $z=0$. DeMaio et al. (2015) show that the ICL is likely to be dominated by stripping from $>0.2 L_{*}$ galaxies rather than stripping or disruption of dwarfs or mergers with the central galaxy. Thus the mass limit is unlikely to have introduced significant biases in the ICL in our simulation.

We then rerun the simulation replacing these haloes by full bulge+disc+DM models as they fall in. This procedure assumes that the star formation of each field galaxy proceeded normally and was then interrupted by ram pressure stripping of the gas upon entering the dense cluster environment (e.g. Peng et al. 2010; Taranu et al. 2014; Tal et al. 2014, and references therein).

Semi-analytic models are very useful for populating systems growing hierarchically (e.g. Moster, Macciò \& Somerville 2014). For our model galaxies to resemble observed galaxies, we select their parameters guided by the semi-analytic model catalogues of Cole et al. (2000). These semi-analytic models employed a 
Table 1. The model galaxies in the simulation.

\begin{tabular}{lccccccccc}
\hline Galaxy & $z_{i}^{a}$ & $\begin{array}{c}M_{200}^{b} \\
\left(10^{10} \mathrm{M}_{\odot}\right)\end{array}$ & $\begin{array}{c}V_{200}^{c} \\
\left(\mathrm{~km} \mathrm{~s}^{-1}\right)\end{array}$ & $\begin{array}{c}M_{*}^{d} \\
\left(10^{10} \mathrm{M}_{\odot}\right)\end{array}$ & $\begin{array}{c}R_{\mathrm{d}}^{e} \\
(\mathrm{kpc})\end{array}$ & $B / D^{f}$ & $R_{e} / R_{\mathrm{d}}^{g}$ & $\begin{array}{c}R_{\mathrm{HL}}^{h} \\
(\mathrm{kpc})\end{array}$ & $M_{V}^{i}$ \\
\hline $\mathrm{G} 01$ & 1.65 & 71.4 & 180 & 4.79 & 2.5 & 0.12 & 0.13 & 3.9 & -19.4 \\
G02 & 1.65 & 12.5 & 106 & 0.74 & 1.1 & 0.27 & 0.17 & 1.5 & -17.4 \\
G03 & 1.13 & 30.4 & 136 & 1.23 & 0.7 & 0.15 & 0.25 & 1.1 & -18.0 \\
G04 & 1.00 & 36.0 & 135 & 1.23 & 0.7 & 0.95 & 0.41 & 0.9 & -17.9 \\
G05 & 1.00 & 28.7 & 127 & 1.13 & 1.7 & 1.11 & 0.31 & 1.8 & -17.8 \\
G06 & 0.81 & 48.9 & 144 & 1.82 & 1.1 & 0.18 & 0.12 & 1.6 & -18.3 \\
G07 & 0.81 & 8.6 & 82 & 0.18 & 0.6 & 0.12 & 0.41 & 1.1 & -15.8 \\
G08 & 0.81 & 117.0 & 190 & 5.69 & 2.5 & 6.84 & 0.44 & 2.3 & -19.5 \\
G09 & 0.58 & 63.2 & 150 & 2.84 & 1.1 & 0.19 & 0.21 & 1.6 & -18.8 \\
G10 & 0.40 & 28.3 & 112 & 0.57 & 2.3 & 0.13 & 0.17 & 3.6 & -17.1 \\
G11 & 0.40 & 35.9 & 121 & 0.82 & 1.3 & 0.13 & 0.13 & 2.0 & -17.3 \\
G12 & 0.40 & 26.1 & 107 & 0.39 & 1.4 & 0.12 & 0.26 & 2.2 & -16.7 \\
G13 & 0.40 & 508.0 & 278 & 13.2 & 2.1 & 0.52 & 0.14 & 2.5 & -20.5 \\
G14 & 0.25 & 21.7 & 95 & 0.27 & 1.9 & 0.17 & 0.26 & 2.9 & -16.3 \\
G15 & 0.25 & 10.1 & 76 & 0.16 & 1.5 & 1.29 & 0.14 & 1.1 & -15.7 \\
G16 & 0.13 & 88.2 & 148 & 2.74 & 2.1 & 0.84 & 1.16 & 3.8 & -18.8 \\
G17 & 0.13 & 11.3 & 72 & 0.05 & 1.1 & 0.57 & 0.76 & 1.8 & -14.4 \\
G18 & 0.13 & 24.3 & 96 & 0.26 & 1.1 & 0.15 & 0.54 & 1.9 & -16.2 \\
\hline
\end{tabular}

${ }^{a}$ Redshift at which the halo is replaced with a full model.

${ }^{b}$ Halo viral mass.

${ }^{c}$ Viral velocity.

${ }^{d}$ Stellar mass.

${ }^{e}$ Disc scalelength.

${ }^{f}$ Bulge-to-disc mass ratio.

${ }^{g}$ Ratio of the bulge effective radius to disc scalelength.

${ }^{h}$ Half-light radius of the galaxy when placed in the simulation.

${ }^{i}$ Absolute magnitude in the $V$-band.

cosmology with $H_{0}=69.7 \mathrm{~km} \mathrm{~s}^{-1} \mathrm{Mpc}^{-1}, \Omega_{0}=0.3, \Omega_{\mathrm{b}}=0.02$ and $\Omega_{\Lambda}=0.70$. Though this semi-analytic model has a lower value of $\Omega_{\mathrm{b}}$ than the concordance cosmology (Bennett et al. 2013), the parameters of the semi-analytic model were tuned to match the observed galaxy properties. As such, sampling galaxy properties from this semi-analytic model is a viable way of selecting structural parameters for galaxies that are replaced. The galaxies in the catalogues were produced at 20 epochs, equally spaced in time between $z=0$ and 6 . Each redshift catalogue contained between $\sim 7100$ and $\sim 8200$ galaxies.

The Cole et al. (2000) catalogues were modelled in a volume of $10^{5} h^{3} \mathrm{Mpc}^{-3}$ and with masses spanning $5 \times 10^{9}$ to $1 \times 10^{15} h^{-1} \mathrm{M}_{\odot}$. The galaxy catalogues generated in this way contain information on the structural properties of the galaxies (masses, sizes, age and metallicity of both disc and halo components) and DM haloes (mass, virial velocity, concentration and spin parameter). In selecting the best galaxy model for each halo, we used the semi-analytic catalogues, choosing the halo that best matches the target $M_{200}$ and $V_{200}$ at the infall redshift; however, we discard matches where the bulge-to-disc mass ratio, $B / D<1 / 9$, which we arbitrarily chose so the spheroid is well populated.

The initial model galaxies were generated using the method of Springel \& White (1999). The models consist of exponential discs, Hernquist bulges (Hernquist 1990) and NFW DM haloes (Navarro, Frenk \& White 1997). In all cases, the vertical profile of the disc is $\operatorname{sech}^{2} z / z_{\mathrm{d}}$, with ratio of scaleheight, $z_{\mathrm{d}}$, to scalelength, $R_{\mathrm{d}}$, set to $z_{\mathrm{d}} / R_{\mathrm{d}}=0.1$. Table 1 lists the initial conditions of the galaxies, including the redshift at which each DM halo is replaced, the halo mass, the circular velocity at $r_{200}$, the stellar mass, the disc scalelength, the bulge-to-disc mass ratio and the ratio of the bulge effective radius to disc scalelength, $R_{\mathrm{e}} / R_{\mathrm{d}}$. We set stellar particle softening to $\epsilon=0.03 R_{\mathrm{d}}$, which ends up corresponding to $\epsilon=34-198 \mathrm{pc}$. We use a larger softening, in the range of $\epsilon=0.57-$ $5.1 \mathrm{kpc}$, for DM particles. In all cases, the discs consist of 300000 particles, while the number of DM particles varies between 301719 and 944783 .

The Hernquist bulges formally extend to large radii. Rather than truncating these bulges at some finite radius, we treat the star particles at large radius as the stellar halo particles. de Jong, RadburnSmith \& Sick (2009) found that bulge and stellar halo density profiles in nearby disc galaxies join smoothly, so it is reasonable to identify the model's outer bulge as the stellar halo, at least from the density profile point of view. However, the actual ratio of stellar halo to disc stars remains model-dependent because the assumed functional form of both the bulge (Hernquist bulge) and the disc (single exponential) are somewhat arbitrary at large radii and certainly difficult to constrain at high redshift. Thus, the ratio of disc to halo stars in the disc outskirts is poorly constrained. Because in general disc density profiles tend to truncate at large radii (e.g. van der Kruit 1979, 1987; Pohlen, Dettmar \& Lütticke 2000; Pohlen et al. 2002; Erwin 2005; Pohlen \& Trujillo 2006), we are very likely overrepresenting the disc contribution to the ICL. The surface brightness profiles for each galaxy as they enter the cluster are presented in Appendix A.

We insert each model galaxy before it crosses the virial radius of the cluster, in order to give the models sufficient time to relax, $>100 \mathrm{Myr}$, since the initial conditions generated using the method of Springel \& White (1999) are not in perfect equilibrium (Kazantzidis et al. 2004).

We compare our galaxies to the observational data of Williams et al. (2010) and the simulations of Laporte et al. (2013) out to redshifts of $z \sim 2$. Fig. 1 shows the size-mass relation of our 18 galaxies when they are first inserted into the simulation, with redshifts between 1.65 and 0.13 and at $z=0$. At $z=0$, we 


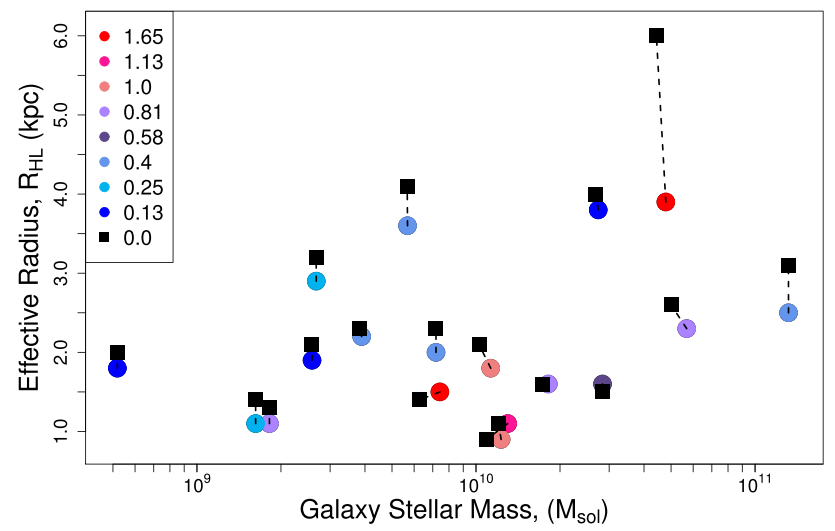

Figure 1. The size-mass relation of our galaxies when inserted into the simulation (circles coloured for redshift) and at $z=0$ (black squares). The dashed lines show the evolution of the mass and stellar size for each galaxy.

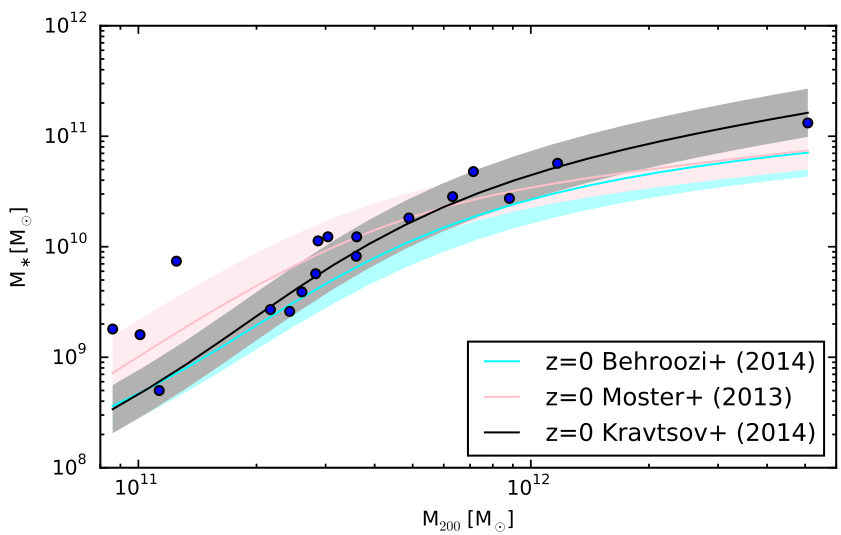

Figure 2. Stellar mass versus halo mass for each resimulated galaxy at the time at which they are introduced (blue points). Assuming these masses do not change if the models were evolved in isolation, we compare with the abundance matching relations from different studies as indicated. The different shaded regions correspond to the uncertainties in each of the relations.

define the half-light radius, $R_{\mathrm{HL}}$, of the galaxies using only stars within our radial cut at $50 \mathrm{kpc}$ (corresponding to the radial cut we use to separate galaxies from ICL described in Section 3.2). Across the redshift range $0<z<2$, our selected galaxies lie within the region of observed galaxies from Newman et al. (2012). The galaxies are slightly less massive than those simulated by Laporte et al. (2013). At redshift slightly higher than the redshift of our first replaced galaxy, the galaxies lie within $1 \sigma$ of the relation of Williams et al. (2010). Therefore our selection of galaxy parameters is realistic. The overall trend revealed by Fig. 1 is for $R_{\mathrm{HL}}$ to increase. Galaxies of stellar mass $M_{*}>5 \times 10^{9} \mathrm{M}_{\odot}$ experience most of the stripping, while lower mass galaxies are not as heavily stripped. Therefore most of the ICL comes from massive galaxies.

As a final check of the model galaxy parameters, we plot in Fig. 2 the stellar mass versus halo mass for each resimulated galaxy at the time at which we introduce it into our $\mathrm{N}$-body simulation. If the galaxies were evolved in isolation, rather than falling into the cluster, we do not believe either of these quantities would change significantly between that time and $z=0$. We therefore compare these initial conditions directly to the $z=0$ abundance matching relations of Behroozi, Wechsler \& Conroy (2013), Moster, Naab \& White (2013) and Kravtsov et al. (2014). Our model galaxies are in

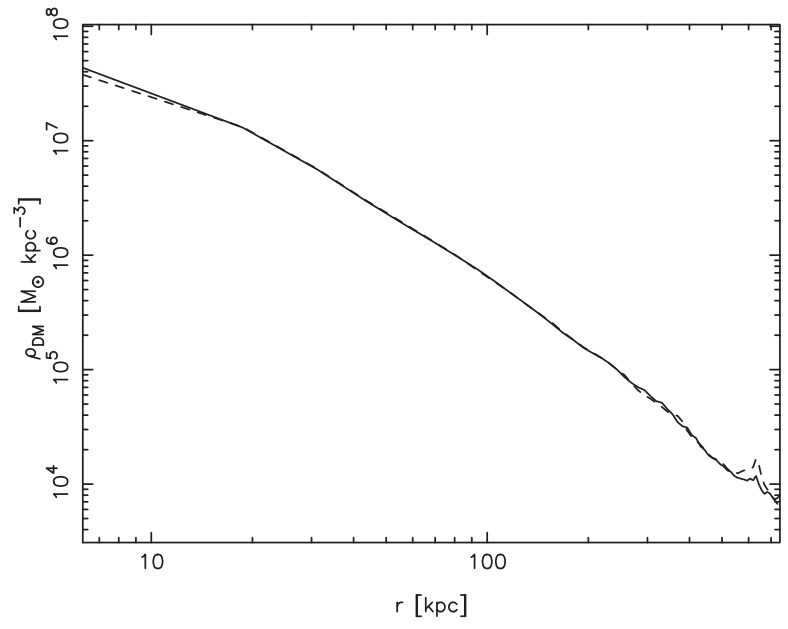

Figure 3. The full DM density profile in the DM-only simulation (solid line) and in our resimulation with infalling haloes replaced (dashed line).

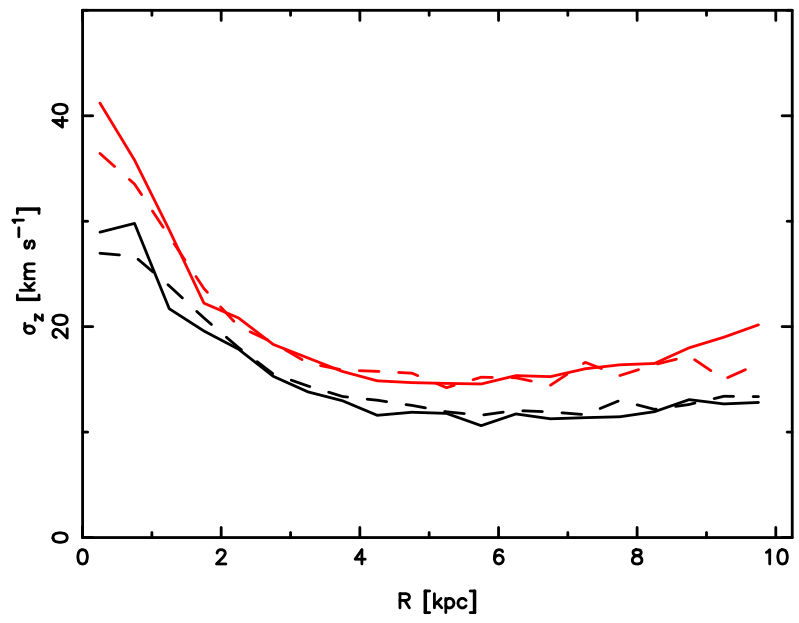

Figure 4. The vertical velocity dispersion of stars, $\sigma_{z}$, at $z=0.24$ (solid lines) and at $z=0$ (dashed lines) for two models that have had a quiet history. The black lines show model G14 while the red lines show model G15.

good agreement with these relations, particularly with the relation of Kravtsov et al. (2014).

\subsection{Tests of the simulation}

We verified that our replacement of infalling DM haloes does not alter the overall structure of the cluster substantially. Fig. 3 shows the density profile of the DM component in the DM-only simulation and in our resimulation with galaxy replacements. It is apparent that our procedure does not alter the cluster.

In order to verify that our simulation is not suffering from excess artificial heating, we use the vertical velocity dispersion of stars, $\sigma_{z}$, (since the in-plane velocity dispersions will increase because of spiral structure). We select two galaxies that have low mass, which are not substantially tidally stripped and that never formed bars, since bars vertically heat discs (e.g. Raha et al. 1991; Debattista et al. 2006): galaxies G14 and G15. These were replaced at $z=0.25$; Fig. 4 shows their vertical velocity dispersions after relaxation, and at the end of the simulation after $\sim 3$ Gyr of evolution. Within $10 \mathrm{kpc}$, $\sigma_{z}$ does not evolve substantially, indicating that the models are not heating because of artificial numerical effects. 


\subsection{Formation of the $\mathrm{cD}$ galaxy}

The stellar component of the $\mathrm{cD}$ galaxy in our simulation is comprised of the stars from only a single galaxy that enters the cluster early and settles to the centre. At $z \simeq 1.65, \mathrm{G} 01$ enters the cluster and undergoes several passes through the cluster centre before finally settling there by $z \simeq 0.6$, thus forming a cD galaxy, at the cluster centre. The $\mathrm{cD}$ is a slowly rotating, pressure-supported, spheroidal system, with a very extended envelope, which are all properties typical of cD galaxies (Oemler 1976; Thuan \& Romanishin 1981; Schombert 1986, 1988; Oegerle \& Hill 1992; Bertola et al. 1995). If we had included material that falls in earlier, when the cluster was more chaotic, then the $\mathrm{cD}$ galaxy would have had a larger stellar mass. However, this mass would have been more centrally concentrated than that from G01 (Diemand et al. 2005), and would therefore contribute a smaller mass fraction to the ICL.

The Fornax cluster's cD galaxy, NGC 1399, has a stellar mass of $2 \times 10^{11} \mathrm{M}_{\odot}$ within $5 \mathrm{kpc}$ (Saglia et al. 2000), compared with the $\mathrm{cD}$ in our simulation, $4.4 \times 10^{10} \mathrm{M}_{\odot}$. The relatively low-mass $\mathrm{cD}$ galaxy in the simulation is a result of the replacements starting from $z=1.65$; indeed at $z=2.7$, we identified an infalling halo with a mass almost twice as that of G01, which seems destined to fall on to the centre of the cluster and contribute to the $\mathrm{cD}$. Besides this, we identified a further seven haloes falling into the cluster between $z=3.8$ and 1.65, with masses spanning from $\sim 9 \times 10^{9}$ to $\sim 1.3 \times 10^{12} \mathrm{M}_{\odot}$. Thus a further five times the mass of the halo of G01 is missed in our replacements. In the rest of this paper, we loosely refer to galaxy $\mathrm{G} 01$ as the $\mathrm{cD}$ galaxy, but it should be remembered that G01 is only a fraction of the total mass that would have gone into the $\mathrm{cD}$ galaxy of a real cluster like Fornax.

\section{DEFINING THE ICL}

\subsection{Imaging the ICL}

We analyse the results of the simulation at $z=0$. To compare with the Fornax cluster, we view the simulation at a similar distance ( $\sim 20 \mathrm{Mpc}$; Blakeslee et al. 2009) and assume a stellar mass-tolight ratio of $\mathrm{M} / \mathrm{L}=5 \mathrm{M}_{\odot} / \mathrm{L} \odot$ (Rudick et al. 2006) to convert the mass to a luminosity. Though this choice is a simplification, this is a characteristic value in the $V$ band for an evolved stellar population at $z=0$, which is the population from which we expect the ICL to be comprised. This $\mathrm{M} / \mathrm{L}$ ratio is important for when we define the ICL at fixed surface brightness but, as we show below, our preferred definition of the ICL depends on distance from individual galaxies and is therefore independent of the assumed $\mathrm{M} / \mathrm{L}$.

We construct a series of cluster images measuring $1500 \times$ 1500 pixels, with each pixel measuring $2 \times 2 \mathrm{kpc}$. This gives a resolution of 0.023 arcsec per pixel compared with Space Telescope Imaging Spectrograph (STIS) imaging on the Hubble Space Telescope, which has 0.05 arcsec per pixel. All of the images are centred on the $\mathrm{cD}$ galaxy. We use three orthogonal projections of the cluster along the $x, y$ and $z$ axes of the simulation, averaging profiles over these three projections. We treat the largest difference between the average and the individual projections as our error estimate. Fig. 5 shows the distribution of all the disc (left-hand side) and halo (right-hand side) stars within the cluster (including both those stars bound in galaxies, and those in the ICL). Stars that originated in the discs are more centrally concentrated. The disc stars are also responsible for long, thin filaments due to them being kinematically cooler populations, which remain more coherent after they are stripped from a galaxy (Rudick et al. 2009).

\subsection{Identifying the ICL}

To identify the ICL, past simulation works have used a $V$-band surface brightness cut of $26.5 \mathrm{mag} \operatorname{arcsec}^{-2}$ (e.g. Rudick et al. 2006, which we term the isophotal method), with everything fainter than this limit being classified as ICL. The isophotal method is also the most commonly used definition of the ICL for observational studies. When applied to our simulation we found this method excluded from the ICL dense areas of tidal streams. Moreover some of the fainter, low surface brightness galaxies were included with the ICL. Likewise Rudick et al. (2011) found that the isophotal method produced less ICL compared to other definitions.

Other methods for identifying the ICL have been suggested, such as binding energy (using halo detection algorithms to find the galaxy potentials and defining the ICL as stars outside these potentials; Dolag et al. 2010) and particle density threshold, which requires knowledge of the number of luminous particles in a given volume. (For more details on these methods, see Rudick et al. 2011.) However, these methods, while useful in simulations, are difficult to apply to observations.

We therefore use a simple radial cut method to remove the galaxies, taking $50 \mathrm{kpc}$ around all the galaxies except galaxy G01 (the $\mathrm{cD}$ ), for which we use a cut-off of $100 \mathrm{kpc}$. These cuts are likely to remove any bound contribution (see the appendix for evidence of this), leaving only the ICL. While the choice of cut may change the ICL fraction by $\sim 50$ per cent, it does not change the overall trends. Our choice of $50 \mathrm{kpc}$ is necessarily arbitrary, with the main requirement being that very few stars still bound to a galaxy are present beyond this radius. We choose twice this radius for galaxy G01 because $\mathrm{cD}$ galaxies usually have extended stellar envelopes (e.g. Oemler 1976; Schombert 1986; Zhao, Aragón-Salamanca \& Conselice 2015), with unclear boundaries (e.g. Rudick et al. 2011). This will classify a smaller fraction of the stellar mass of the cluster as the ICL, but will be more robust to misclassification of faint galaxies as ICL, while being easy to implement observationally.

Nonetheless, for comparison, we have also carried out our analysis using a surface brightness cut-off at $26.5 \mathrm{mag} \operatorname{arcsec}^{-2}$. Our radial cut method leads to roughly twice the amount of ICL as produced by a surface brightness cut $(\sim 4$ percent using a radial cut versus $\sim 2$ per cent using a surface brightness cut) and the fractional difference is largest for disc stars, where fewer stars are classified as ICL by the surface brightness cut (by roughly a factor of 4 , whereas the difference is a factor of 3 for bulge stars). In real observations, a cut of $50 \mathrm{kpc}$ would be too extreme if it was applied to every galaxy, including the dwarfs, which we have not replaced.

\section{RESULTS}

\subsection{ICL luminosity}

\subsubsection{ICL fraction}

Table 2 lists the fraction of each galaxy that is stripped from the galaxy and becomes ICL. In general, the galaxies that have contributed the greatest percentage of their stellar mass to the ICL are the ones that fell in earliest. Very little mass is lost to the ICL by galaxies that fall into the cluster after $z=0.6$, regardless of stellar mass.

Of the total stellar mass included in the $\mathrm{N}$-body galaxy models $\left(3.81 \times 10^{11} \mathrm{M}_{\odot}\right)$, we classify 4.4 per cent $\left(1.65 \times 10^{10} \mathrm{M}_{\odot}\right)$ as ICL. When calculating the fraction of the cluster in the ICL, we use the total cluster mass (i.e. including the mass still bound to other 

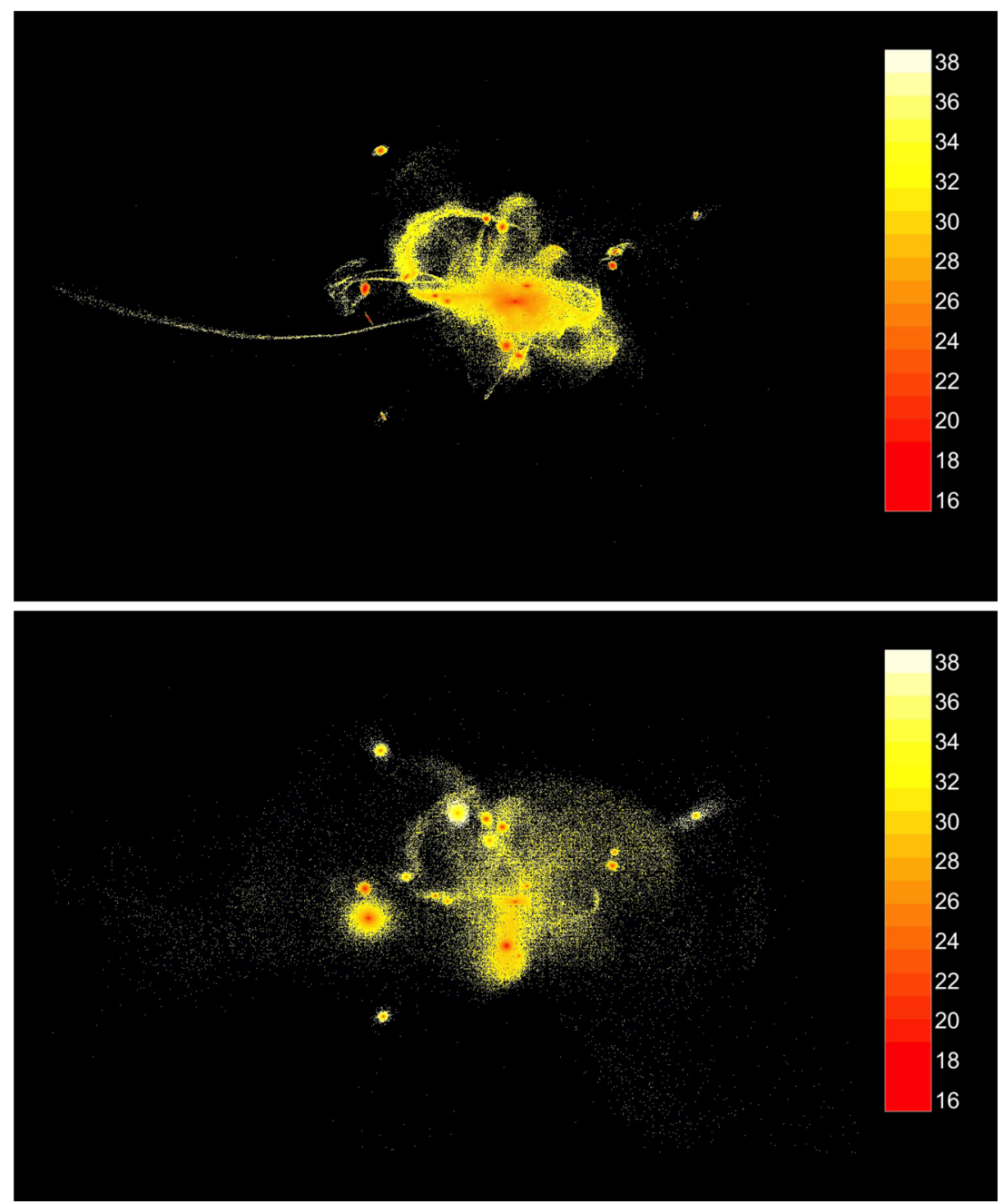

Figure 5. The surface brightness (in the $V$ band) distribution of the disc (top panel) and halo (bottom panel) stars within the cluster. Disc stars are more centrally concentrated, and exhibit more conspicuous narrow tidal features.

cluster members and the unbound mass) as the total mass. This definition has been used throughout this paper. Using the radial cut, 51 per cent of the ICL comes from disc stars, while the rest is from haloes; if we exclude G01's (the central galaxy) contribution to the ICL, the disc fraction is 38 percent of the remaining ICL. These have been in the cluster longer, are more centrally concentrated within the cluster and therefore have been more disrupted than the younger galaxies. It should be noted that the total stellar mass of the cluster as defined by an observer is likely to be greater than the mass described in this $\mathrm{N}$-body simulation, as the simulation does not resimulate all the galaxies that fall into the cluster, nor a large fraction of the stars in the $\mathrm{cD}$ galaxy. Since smaller galaxies and the $\mathrm{CD}$ would presumably contribute more to the total mass of the cluster than they would to the ICL, the ICL fraction would presumably decrease if they were included.

\subsubsection{Surface brightness distribution}

Fig. 6 shows the distribution of the surface brightnesses of the ICL and its contributions from the disc and halo stars. The stellar halo distribution peaks at a surface brightness $\sim 27 \mathrm{mag} \mathrm{arcsec}^{-2}$, while the disc stellar distribution peaks at a brighter surface brightness of $\sim 25 \mathrm{mag} \operatorname{arcsec}^{-2}$. It is clear from this figure that the isophotal cut method is too draconian, as it removes a significant number of originally disc stars from the ICL.

\subsection{3 cD galaxy contribution}

Table 3 presents the percentages that the galaxy G01 (the cD) and its components contribute to both the total cluster luminosity and to the ICL. G01 contributes 22 percent of the stars in the ICL (similar to the result in Murante et al. 2007). The majority of these stars originate from the disc of G01. Only 0.5 percent of the ICL is made up of halo stars from G01, even though for this galaxy, the initial bulge to disc ratio is 0.12 . The contribution from G01 to the ICL is shown in Fig. 6. As the orbit of G01 decays to the centre, it makes multiple passes through the cluster centre and is therefore strongly disrupted spreading the stars from the progenitor over a large volume.

\subsection{ICL radial profile}

Fig. 7 shows the radial profile of the ICL. Close to the centre of the cluster, disc stars make up the greater part of the ICL, dominated by those from G01 (the cD). Beyond $\sim 200 \mathrm{kpc}$, the halo stars become the main source of the ICL. When the 
Table 2. The values for each galaxy with the initial mass, mass lost to the ICL, the percentage of the ICL contributed from each galaxy (broken into disc and bulge stars).

\begin{tabular}{|c|c|c|c|c|c|c|c|c|}
\hline Galaxy & $z_{i}^{a}$ & $\begin{array}{c}\text { Initial galaxy } \\
\text { stellar mass } \\
\left(10^{10} \mathrm{M}_{\odot}\right)\end{array}$ & $\begin{array}{c}\text { Galaxy stellar } \\
\text { mass at } z=0 \\
\left(10^{10} \mathrm{M}_{\odot}\right)\end{array}$ & $\begin{array}{c}\text { ICL mass } \\
\text { at } z=0 \\
\left(\mathrm{M}_{\odot}\right)\end{array}$ & $\begin{array}{l}\text { Per cent of mass } \\
\text { lost to ICL }\end{array}$ & Per cent of ICL & $\begin{array}{l}\text { Per cent of ICL } \\
\text { from bulge stars }\end{array}$ & $\begin{array}{l}\text { Per cent of ICL } \\
\text { from disc stars }\end{array}$ \\
\hline G02 & 1.65 & 0.74 & 0.63 & $1.2 \times 10^{9}$ & 18.2 & 6.95 & 0.56 & 6.50 \\
\hline G03 & 1.13 & 1.30 & 1.09 & $2.1 \times 10^{9}$ & 19.2 & 12.7 & 0.83 & 11.86 \\
\hline G04 & 1.0 & 1.23 & 1.20 & $2.5 \times 10^{8}$ & 2.09 & 1.53 & 1.52 & 0.008 \\
\hline G05 & 1.0 & 1.13 & 1.03 & $1.0 \times 10^{9}$ & 10.2 & 6.31 & 3.00 & 3.31 \\
\hline G07 & 0.81 & 0.18 & 0.18 & $3.3 \times 10^{6}$ & 0.18 & 0.02 & 0.02 & 0.0003 \\
\hline G08 & 0.81 & 5.69 & 5.00 & $6.9 \times 10^{9}$ & 13.8 & 41.75 & 39.1 & 2.67 \\
\hline G09 & 0.58 & 2.84 & 2.84 & $6.2 \times 10^{6}$ & 0.022 & 0.037 & 0.032 & 0.005 \\
\hline G10 & 0.4 & 0.57 & 0.567 & $1.4 \times 10^{7}$ & 0.25 & 0.086 & 0.018 & 0.068 \\
\hline G11 & 0.4 & 0.72 & 0.72 & $3.1 \times 10^{6}$ & 0.044 & 0.019 & 0.003 & 0.016 \\
\hline G12 & 0.4 & 0.39 & 0.38 & $6.4 \times 10^{7}$ & 1.66 & 0.39 & 0.06 & 0.32 \\
\hline G18 & 0.13 & 0.26 & 0.26 & $4.1 \times 10^{5}$ & 0.016 & 0.003 & 0.003 & 0.0 \\
\hline
\end{tabular}

${ }^{a}$ Redshift at which the galaxy is inserted into the cluster.

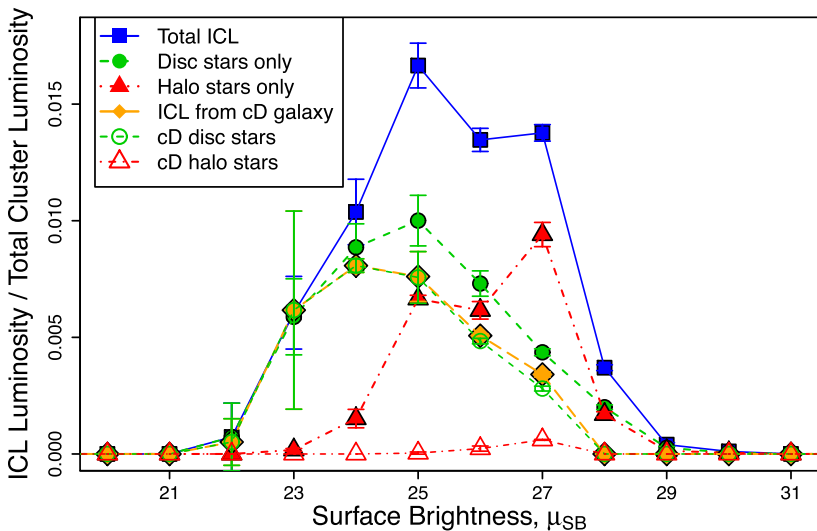

Figure 6. The ICL luminosity fraction as a function of surface brightness (see the key for symbols.) The change from a disc-dominated ICL to a halodominated ICL occurs at $\sim 26 \mathrm{mag} \operatorname{arcsec}^{-2}$ in the $V$ band. Each pixel is $2 \times 2 \mathrm{kpc}$, corresponding to 0.023 arcsec per pixel at the distance of Fornax.

contribution of G01 is removed, at all radii the majority of the ICL is comprised of halo stars. This is because, further out in the cluster, only the less gravitationally bound halo stars are stripped from galaxies.

Fig. 8 shows the profile of the mass density as a function of radius for the ICL, ICL disc stars and ICL halo stars, compared with the DM density profile. The ICL stars have a steeper density profile, including within $r_{200} / 2$, than the DM. The steeper falloff of baryons relative to the DM is expected, since the density profiles of stellar haloes also tend to drop off more rapidly than those of the DM haloes (e.g. Mandelbaum et al. 2010).

Fig. 9 shows the ratio of the halo-to-disc stars for the whole of the ICL, and with the contribution of G01 (cD) excluded. G01 only has a minimal effect on the ICL halo component and contributes little ICL beyond a radius of $\sim 250 \mathrm{kpc}$. At distances $R>350 \mathrm{kpc}$, no stars from G01 (disc or halo) are present in the ICL.

\subsection{Stellar ages}

The galaxies entered the cluster at different times. When the galaxies fall into the cluster, we assume they cease star formation so the stellar age depends on the initial age of the stars when the galaxy enters the cluster. The semi-analytic models provide us with stellar ages for the bulges and discs of each galaxy. Generally, the disc stars are younger than the halo stars. Fig. 10 shows the ages of halo ICL and disc ICL stars and the average age of the ICL as a function of cluster radius. Since halo ICL stars are more prevalent at $R>200 \mathrm{kpc}$ (shown in Fig. 7), the mean stellar age will be older further from the cluster centre. Fig. 10 also shows the average age of the stars in the galaxies. (The errors on the galaxy distance and stellar age are derived from the variation in orientation.) The galaxies themselves exhibit a steeper average age profile than does the ICL; this is a reflection of the early formation of the ICL and the later infall of the cluster galaxies, which remain at large radii. As younger galaxies lie further out from the cluster centre than the older galaxies, they experience less disruptions and lose fewer stars to the ICL. Therefore the ICL consists mainly of stars removed from the haloes of older cluster members.

\section{DISCUSSION AND CONCLUSIONS}

We have presented a collisionless simulation studying the ICL in a Fornax-cluster-mass system. For our simulated cluster, we estimate an ICL fraction of $\sim 4$ per cent of the cluster stellar mass. This value is within the range of most of the observations, though somewhat lower than simulated values (e.g. Sommer-Larsen, Romeo \& Portinari 2005; Puchwein et al. 2010). If we include G01 into the ICL, this fraction becomes $\sim 16$ per cent. Table 4 compares the ICL fraction in our simulation with that obtained by a number of observational and theoretical studies.

In this mass regime, the majority of the ICL outside G01 (the $\mathrm{cD}$ galaxy) is derived from the haloes of galaxies: $\sim 51$ per cent of its mass from stars that started their life in galactic discs $(\sim 38$ per cent if G01 is excluded). Since our starting models have extended pure 
Table 3. Percentages of the cluster luminosity contained in the ICL, and the ICL disc and halo stars from the central (cD) galaxy G01.

\begin{tabular}{lccc}
\hline Method & All ICL from G01 & ICL disc stars from G01 & ICL halo stars from G01 \\
\hline Contribution to total galaxy cluster & 0.95 per cent \pm 0.21 percent & 0.92 per cent \pm 0.20 per cent & 0.03 percent \pm 0.012 percent \\
Contribution to the ICL & 21.9 per cent \pm 6.5 per cent & 21.3 per cent \pm 6.3 per cent & 0.54 percent \pm 0.03 per cent \\
\hline
\end{tabular}

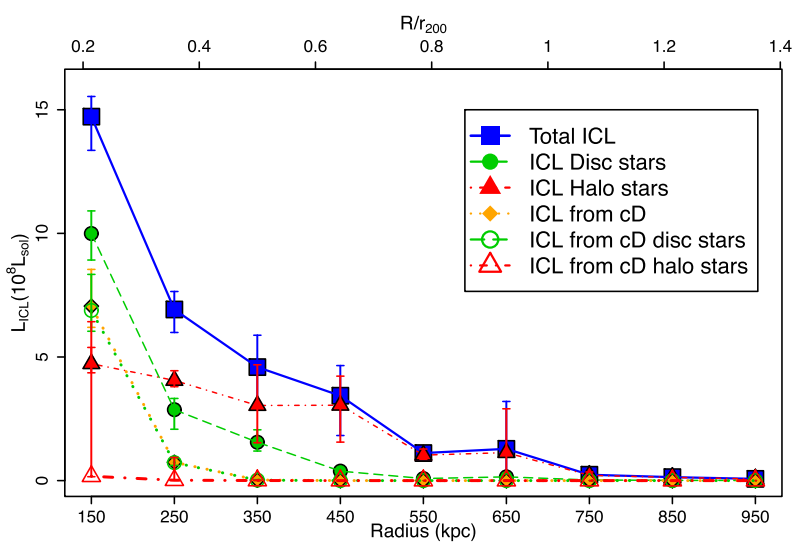

Figure 7. The average ICL luminosity contribution as a function of radius for the different components. The change from disc- to halo-dominated ICL occurs at $\sim 200 \mathrm{kpc}$. The contribution from the central $(\mathrm{cD})$ galaxy also declines rapidly with radius and does not contribute any ICL beyond $R \sim 350 \mathrm{kpc}$.

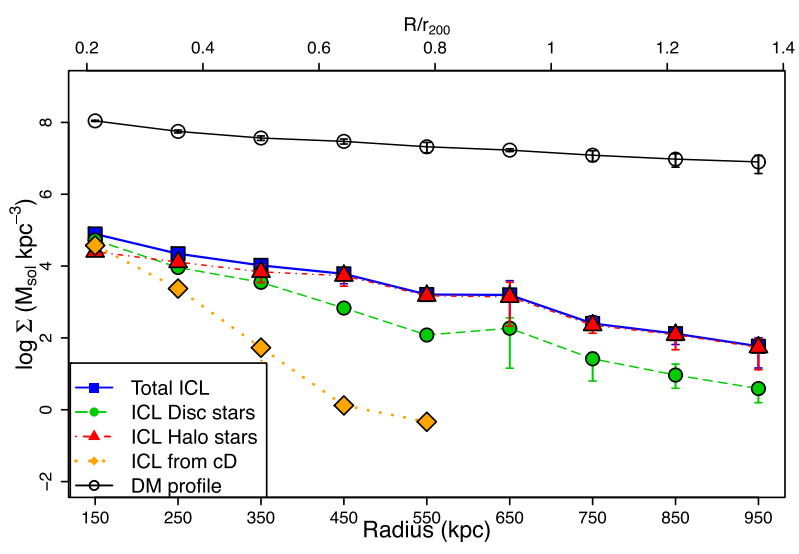

Figure 8. The mass density profile as a function of cluster radius for the ICL, disc ICL stars, halo ICL stars, ICL from G01 (cD) and the DM profile.

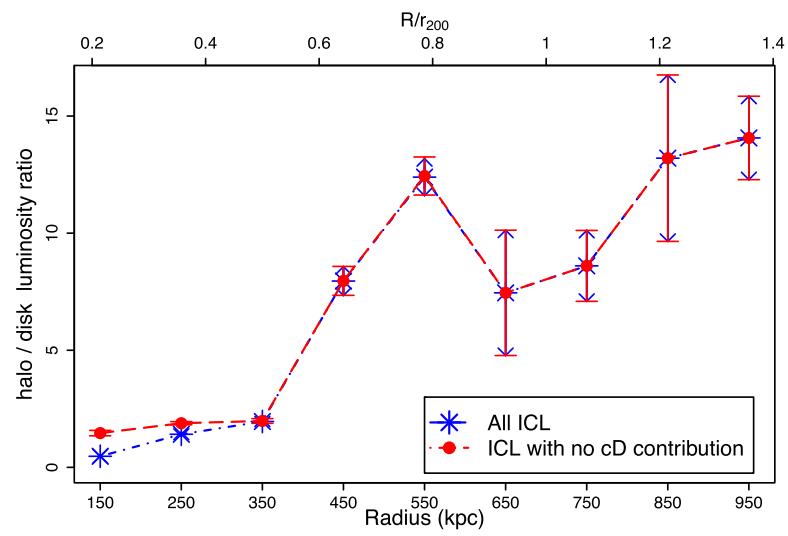

Figure 9. The ratio of the halo-to-disc stars in the ICL as a function of radius from the centre of the cluster for the ICL as a whole (red circles, dashed line) and with the contribution from G01 (cD) removed (blue asterisks, dot-dashed line).

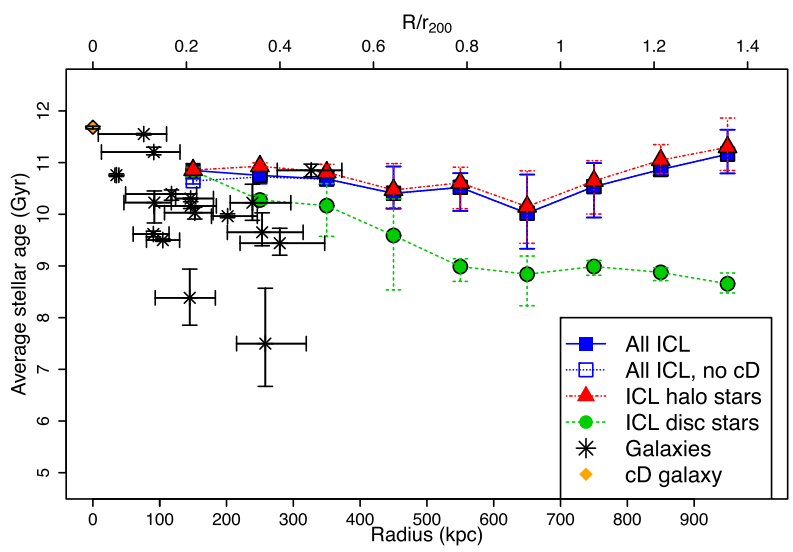

Figure 10. The average age of disc and halo ICL stars as a function of radius. (See the key for the symbols.) The plot also shows the ages with and without the contribution of G01 (the cD). The disc stars are, on average, younger than the halo stars and the difference in ages increases at larger radii. The average age of the galaxies is also indicated.

exponential density profiles to large radii, whereas the majority of real disc galaxies are truncated, the amount of stellar mass in the simulation's outer discs is overestimated. This gives us confidence that our disc ICL fraction is an upper limit. However, inside $0.25 r_{200}$, the ICL is dominated by stars from the disc of G01, the galaxy that becomes part of the $\mathrm{cD}$ (see Fig. 7).

We studied the contribution to the ICL from individual galaxies and found that the largest, oldest galaxies contribute the most to the ICL. This agrees with Contini et al. (2014) who found that 26 per cent of ICL comes from galaxies with stellar masses between $10^{10.75}$ and $10^{11.25} \mathrm{M}_{\odot}$ and 68 per cent of ICL is from galaxies with $M_{*}>10^{10.5} \mathrm{M}_{\odot}$. For galaxies of mass $M_{*}<4 \times 10^{9} \mathrm{M}_{\odot}$, their contribution to the total ICL is negligible. We note that this is close to our cut-off for the replaced galaxies. Thus it is reasonable to suppose that we have not missed a significant fraction of the ICL from low-mass galaxies in our simulation.

The radial distribution of the ICL shows a change in composition from being disc-dominated near the cluster centre to stellar halodominated by $\sim 25$ percent of $r_{200}$ (Fig. 7). This change is due to G01 contributing the majority of the disc ICL stars. The distance is approximately half the distance found by Murante et al. (2007) for a cluster of virial mass $1.6-2.9 \times 10^{14} h^{-1} \mathrm{M}_{\odot}$.

The stellar ages are different between the galaxies and the ICL as a function of radius. The older galaxies within the cluster tend to lie near the cluster centre while the younger galaxies lie further out, which reflects the time of infall into the cluster. ICL stars are, on average, older than the average galaxy stars, particularly at large radii, with the average age nearly constant or increasing slightly with radius. This is similar to the result found by Puchwein et al. (2010). The radially nearly constant age of the ICL stars is due to most of them having been stripped from galaxies that fell into the cluster at an early epoch. The younger galaxies are not as heavily stripped so do not have as large an impact on the ICL. This old ICL is different from the semi-analytic model result of Contini et al. (2014), who find a predominantly younger ICL (forming since $z=1$ ). 
Table 4. Comparison of the ICL fraction in our simulation and in observed, simulated and semi-analytic model clusters.

\begin{tabular}{|c|c|c|c|c|c|}
\hline & $\begin{array}{c}\text { Cluster mass } \\
\quad\left(\mathrm{M}_{\odot}\right)\end{array}$ & ICL fraction & $\mathrm{ICL}+\mathrm{BCG}$ & Reference & $\begin{array}{l}\text { ICL detection } \\
\text { and notes }\end{array}$ \\
\hline \multirow[t]{5}{*}{ Observations } & $1.4 \times 10^{15}$ & $5.9 \pm 1.8$ percent & $8.2 \pm 2.5$ percent & Presotto et al. (2014) & Source subtraction $M\left(R<R_{500}\right)$ \\
\hline & $10^{13}-10^{14}$ & $2.69 \pm 1.6$ & & McGee \& Balogh (2010) & Hostless $\mathrm{SNe}$ rates \\
\hline & $10^{13}-10^{14}$ & $15.8 \pm 8$ & & Feldmeier et al. (2004a) & Isophotal cut-off (non-cD clusters) \\
\hline & $\sim 10^{14}$ & $1-4$ per cent & & Burke et al. (2012) & Isophotal cut-off (at $z \sim 1$ ) \\
\hline & & $7-15$ per cent & & Feldmeier et al. (2004b) & Planetary nebulae (Virgo cluster) \\
\hline & & & & & Methods for separating ICL from BCG \\
\hline & $10^{14}$ & 9-36 per cent & & Rudick et al. (2011) & Binding energy/double-Gaussian \\
\hline & & & & & Kinematic fit/density cut-off \\
\hline & $10^{13}-10^{14}$ & $21-34$ per cent & & Sommer-Larsen et al. (2005) & Stars outside the tidal \\
\hline & & & & & Radii of all galaxies \\
\hline & $>10^{13}-10^{15}$ & 20-40 per cent & & Contini et al. (2014) & SAM assuming stripped stars \\
\hline
\end{tabular}

Our simulation does not include gas; nonetheless we produce an ICL fraction comparable to that observed (e.g. Feldmeier et al. 2004b; Burke et al. 2012). Hydrodynamical simulations instead have produced much higher ICL fractions (e.g. Sommer-Larsen et al. 2005; Puchwein et al. 2010; Cui, Borgani \& Murante 2014), some of it from stars forming directly out of cluster gas (Puchwein et al. 2010). On the other hand, the ages of galaxies in our simulation are similar to those of Puchwein et al. (2010). The main assumption upon which the ages of our galaxies rest, that star formation is quenched upon the galaxies entering the cluster, is borne out by the fully cosmological simulations.

In our simulation, the ICL density profile falls off more rapidly than that of the DM. That the baryons are more centrally concentrated than the DM is not surprising since they lose energy in settling to the centre of haloes (White \& Rees 1978).

\section{ACKNOWLEDGEMENTS}

$\mathrm{KAH}$ would like to thank the University of Central Lancashire for the grant to start this work, and Virginia Tech for allowing its completion, also John Feldmeier for his discussion. VPD is supported by STFC Consolidated grant \#ST/J001341/1. VPD, AJC and BBT would like to acknowledge the Undergraduate Research Internship Scheme (2011) for the funding to support early analysis of this simulation, and also the Nuffield Foundation (2011) undergraduate bursary scheme and the Royal Astronomical Society (2012) for the support that contributed to the initial analysis in this paper. EWP acknowledges support from the National Natural Science Foundation of China under Grant No. 11173003, and from the Strategic Priority Research Programme, 'The Emergence of Cosmological Structures', of the Chinese Academy of Sciences, Grant No. XDB09000105. The authors acknowledge the Texas Advanced Computing Center (TACC) ${ }^{1}$ at The University of Texas at Austin for providing HPC resources that have contributed to the research results reported within this paper. We thank Sam Earp for preparing Fig. 2 for us.

\section{REFERENCES}

Aguerri J. A. L., Gerhard O. E., Arnaboldi M., Napolitano N. R., CastroRodriguez N., Freeman K. C., 2005, AJ, 129, 2585

\footnotetext{
${ }^{1}$ http://www.tacc.utexas.edu
}

Arnaboldi M., 2004, in Duc P.-A., Braine J., Brinks E., eds, Proc. IAU Symp. 217, Intracluster Stellar Population. Kluwer, Dordrecht, p. 54

Arnaboldi M., 2005, in Szczerba R., Stasińska G., Gorny S. K., eds, AIP Conf. Proc. Vol., Intracluster Planetary Nebulae as Dynamical Probes of the Diffuse Light in Galaxy Clusters. Am. Inst. Phys., New York, p. 301

Arnaboldi M., Gerhard O., 2010, Highlights Astron., 15, 97

Arnaboldi M. et al., 1996, ApJ, 472, 145

Behroozi P. S., Wechsler R. H., Conroy C., 2013, ApJ, 770, 57

Bennett C.L et al., 2013, ApJS, 208, 20

Bertola F., Cinzano P., Corsini E. M., Rix H.-W., Zeilinger W. W., 1995, ApJ, 448, 13

Binggeli B., Popescu C. C., Tammann G. A., 1993, A\&AS, 98, 275

Blakeslee J. P. et al., 2009, ApJ, 694, 556

Burke C., Collins C. A., Scott J. P., Hilton H., 2012, MNRAS, 425, 2058

Calcáneo-Roldán C., Moore B., Bland-Hawthorn J., Malin D., Sadler E. M., 2000, MNRAS, 314, 324

Castro-Rodriguéz N., Arnaboldi M., Aguerri J. A. L., Gerhard O., Okamura S., Yasuda N., Freeman K. C., 2009, A\&A, 507, 621

Churazov E., Forman W., Vikhlinin A., Tremaine S., Gerhard O., Jones C., 2008, MNRAS, 388, 1062

Cole S., Lacey C. G., Baugh C. M., Frenk C. S., 2000, MNRAS, 319, 168

Conroy C., Gunn J. E., 2007, ApJ, 712, 833

Contini E., De Lucia G., Villalobos Á., Borgani S., 2014, MNRAS 437, 3787

Cooper A. P., Gao L., Quo Q., Frenk C. S., Jerkins A., Springel V., White S. D. M., 2015, MNRAS, 451, 2703

Cora S. S., 2006, MNRAS, 368, 1540

Cui W., Borgani S., Murante G., 2014, MNRAS, 441, 1769

Davies J. I. et al., 2013, MNRAS, 428, 834

de Jong R. S., Radburn-Smith D. J., Sick J. N., 2009, in Andersen J., BlandHawthorn J., Nordström B., eds, Proc. IAU Symp. 254, The Galaxy Disk in Cosmological Context. Cambridge Univ. Press, Cambridge, p. 19

Debattista V. P., Mayer L., Carollo C. M., Moore B., Wadsley J., Quinn T., 2006, ApJ, 645, 209

DeMaio T., Gonzalez A. H., Zabludoff A., Zaritsky D., Bradač M., 2015, MNRAS, 448, 1162

Diemand J., Madau P., Moore B., 2005, MNRAS, 364, 367

Dolag K., Murante G., Borgani S., 2010, MNRAS, 405, 1544

Drinkwater M. J. et al., 2000, A\&A, 355, 900

Drinkwater M. J., Gregg M. D., Colless M., 2001, ApJ, 548, 139

Durrell P. R., Ciardullo R., Feldmeier J. J., Jacoby G. H., Sigurdsson S., 2002, ApJ, 570, 119

Durrell P. R. et al., 2014, ApJ, 794, 103

Erwin P., 2005, MNRAS, 364, 283

Feldmeier J. J., Mihos J. C., Morrison H. L., Rodney S. A., Harding P., 2002, ApJ, 575, 779 
Feldmeier J. J., Ciardullo R., Jacoby G. H., Durrell P. R., 2003, ApJS, 145, 65

Feldmeier J. J., Mihos J. C., Morrison H. L., Harding P., Kaib N., Dubinski J., 2004a, ApJ, 609, 617

Feldmeier J. J., Ciardullo R., Jacony G. H., Durrell P. R., Mihos J. C., 2004b, ApJ, 615, 196

Ferguson C., Tanvir N. R., von Hippel T., 1998, Nature, 391, 461

Forman W., Jones C., 1990, in Oegerle W. R., Fitchett J., Danly L., eds, Clusters of Galaxies. Cambridge Univ. Press, Cambridge, p. 257

Fouqué P., Solanes J. M., Sanchis T., Balkowski C., 2001, A\&A, 375, 770

Ftaclas C., Struble M. F., Fanelli M. N., 1984, ApJ, 282, 19

Gal-Yam A., Maoz D., Guhathakurta P., Filippenko A. V., 2003, AJ, 125, 1087

Giallongo E. et al., 2014, ApJ, 781, 24

Gonzalez A. H., Zabludoff A. I., Zaritsky D., 2005, ApJ 618, 195

Gonzalez A. H., Zaritsky D., Zabludoff A. I., 2007, ApJ 666, 147

Guennou L. et al., 2012, A\&A, 537, 64

Hernquist L., 1990, ApJ, 356, 359

Hilz M., Naab T., Ostriker J. P., Thomas J., Burkert A., Jesseit R., 2012, MNRAS, 425, 3119

Hilz M., Naab T., Ostriker J. P., 2013, MNRAS, 429, 2924

Ikebe Y. et al., 1992, ApJ, 384, 5

Jee M. J., 2010, ApJ, 717, 420

Katz N., White S. D. M., 1993, ApJ, 412, 455

Kazantzidis S., Kravtsov A. V., Zentner A. R., Allgood B., Nagai D., Moore B., 2004, ApJ, 611, L73

Kim S. et al., 2014, ApJS, 215, 22

Kravtsov V., Alcaíno G., Marconi G., Alvarado F., 2014, ApJ, 783, 56

Laporte C. F. P., White S. D. M., Naab T., Gao L., 2013, MNRAS, 435, 901

Lee J., Kim S., Rey S. C., 2015, ApJ, 807, 122

Lin Y. T., Mohr J. J., 2004, ApJ, 617, 879

McGee S. L., Balogh M. L., 2010, MNRAS, 403, 79

Mandelbaum R., Seljak U., Baldauf T., Smith R. E., 2010, MNRAS, 405, 2078

Martel H., Barai P., Brito W., 2012, ApJ, 757, 48

Melnick J., Giraud E., Toledo I., Selman F., Quintana H., 2012, MNRAS, 427,850

Merritt D., 1984, ApJ, 276, 26

Mihos J. C., Harding P., Feldmeier J., Morrison H., 2005, ApJ, 631, 41

Mihos J. C., Janowiecki S., Feldmeier J. J., Harding P., Morrison H., 2009, ApJ, 698, 1879

Moore B., Katz N., Lake G., Dressler A., Oemler A., 1996, Nature, 379, 613

Moster B. P., Naab T., White S. D. M., 2013, MNRAS, 428, 312

Moster B. P., Macciò A. V., Somerville R. S., 2014, MNARS, 437, 1027

Murante G. et al., 2004, ApJ, 607, 83

Murante G., Giovalli M., Gerhard O., Arnaboldi M., Borgani S., Dolag K., 2007, MNRAS, 377, 2

Napolitano N. R. et al., 2003, ApJ, 594, 172

Nasonova O. G., de Freitas Pacheco J. A., Karachentsev I. D., 2011, A\&A, 532,104

Navarro J. F., Frenk C. S., White S. D. M., 1997, ApJ, 490, 493

Neill J. D., Shara M. M., Oegerle W. R., 2005, ApJ, 618, 692

Newman A. B., Ellis R. S., Bundy K., Treu T., 2012, ApJ, 746, 162

Oegerle W. R., Hill J. M., 1992, AJ, 104, 2078

Oemler A., Jr, 1976, ApJ, 209, 693

Palladino L. E. et al., 2012, AJ, 143, 128

Peñarrubia J., Ma Y.-Z., Walker M. G., McConnachie A., 2014, MNRAS, 443, 2204

Peng Y.-j. et al., 2010, ApJ, 721, 193

Peng E. W. et al., 2011, ApJ, 730, 23

Peterson J. R., Kahn S. M., Paerels F. B. S., Kaastra J. S., Tamura T., Bleeker J. A. M., Ferrigno C., Jernigan J. G., 2003, ApJ, 590, 207

Pohlen M., Trujillo I., 2005, A\&A, 454, 759

Pohlen M., Dettmar R.-J., Lütticke R., 2000, A\&A 357, 1
Pohlen M., Dettmar R.-J., Lütticke R., Aronica G., 2002, A\&A 392, 807

Presotto V. et al., 2014, A\&A, 565, A126

Puchwein E., Springel V., Sijacki D., Dolag K., 2010, MNRAS, 406, 936

Purcell C. W., Bullock J. S., Zentner A. R., 2007, ApJ, 666, 20

Raha N., Sellwood J. A., James R. A., Kahn F. D., 1991, Nature, 352, 411

Rudick C. S., Mihos J. C., McBride C. K., 2006, ApJ, 648, 936

Rudick C. S., Mihos J. C., Frey L. H., McBride C. K., 2009, ApJ, 699, 1518

Rudick C. S., Mihos J. C., McBride C. K., 2011, ApJ, 732, 48

Saglia R. P., Kronawitter A., Gerhard O., Bender R., 2000, ApJ, 119, 153

Sand D. J. et al., 2011, ApJ, 729, 142

Schombert J. M., 1986, ApJS, 60, 603

Schombert J. M., 1988, ApJ, 328, 475

Shara M. M., 2006, AJ, 131, 2980

Sivanandam S., Zabludoff A. I., Zaritsky D., Gonzalez A. H., Kelson D. D., 2009, ApJ, 691, 1787

Smith H. A., 1981, AJ, 86, 998

Sommer-Larsen J., Romeo A. D., Portinari L., 2005, MNRAS, 357, 478

Spergel D. N. et al., 2003, ApJS, 148, 175

Springel V., White S. D. M., 1999, MNRAS, 307, 162

Stadel J. G., 2001, PhD thesis, Univ. Washington

Sun M., Donahue M., Roediger E., Nulsen P. E. J., Voit G. M., Sarazin C., Forman W., Jones C., 2010, ApJ, 708, 946

Tal T. et al., 2014 ApJ, 789, 164

Taranu D. S., Hudson M. J., Balogh M. L., Smith R. J., Power C., Oman K. A., Krane B., 2014, MNRAS, 440, 1934

Thuan T. X., Romanishin W., 1981, ApJ, 248, 439

Toledo I., Melnick J., Selman F., Quintana H., Giraud E., Zelaya P., 2011, MNRAS, 414, 602

van der Kruit P. C., 1979, A\&A, 38, 15

van der Kruit P. C., 1987, A\&A, 173, 59

White S. D. M., 1976, MNRAS, 174, 19

White S. D. M., 1978, MNRAS, 184, 185

White S. D. M., Rees M. J., 1978, MNRAS, 183, 341

Williams B. F. et al., 2007, ApJ, 654, 835

Williams R. J., Quadri R. F., Franx M., van Dokkum P., Toft S., Kriek M., Labbé I., 2010, ApJ, 713, 738

Willman B., Governato F., Wadsley J., Quinn T., 2004, MNRAS, 355, 159

Zhang Y.-Y, Laganá T. F., Pierini D., Puchwein E., Schneider P., Reiprich T. H., 2011, A\&A, 535, 78

Zhao D., Aragón-Salamanca A., Conselice C. J., 2015, MNRAS, 453, 4444

Zibetti S., 2008, in Davies J. I, Disney M. J., eds, Proc. IAU Symp. 244, Dark Galaxies and Lost Baryons. Kluwer, Dordrecht, p. 176

Zibetti S., White S. D. M., 2005, in Jerjen H., Binggeli B., Proc. IAU Colloq. 198, Near-fields Cosmology With Dwarf Elliptical Galaxies. Cambridge Univ. Press, Cambridge, p. 337

Zwicky F., 1951, PASP, 63, 61

\section{SUPPORTING INFORMATION}

Supplementary data are available at MNRAS online.

Figure A1. Dark matter volume density profile for G01-G06.

Figure A2. Surface brightness profile in the $V$ band for the whole galaxy (black solid), the disc stars (blue dotted) and the bulge stars. Figure A3. The orbit taken by galaxies G01-G06. The initial insertion point is given by a brown diamond.

Please note: Oxford University Press is not responsible for the content or functionality of any supporting materials supplied by the authors. Any queries (other than missing material) should be directed to the corresponding author for the article. 


\section{APPENDIX A: DENSITY PROFILES, DARK MATTER VOLUME PROFILES AND ORBITS OF THE INSERTED MODEL GALAXIES}

Fig. A1 shows the dark matter volume density profile for all the models. The solid line is the halo at the time of insertion, and the
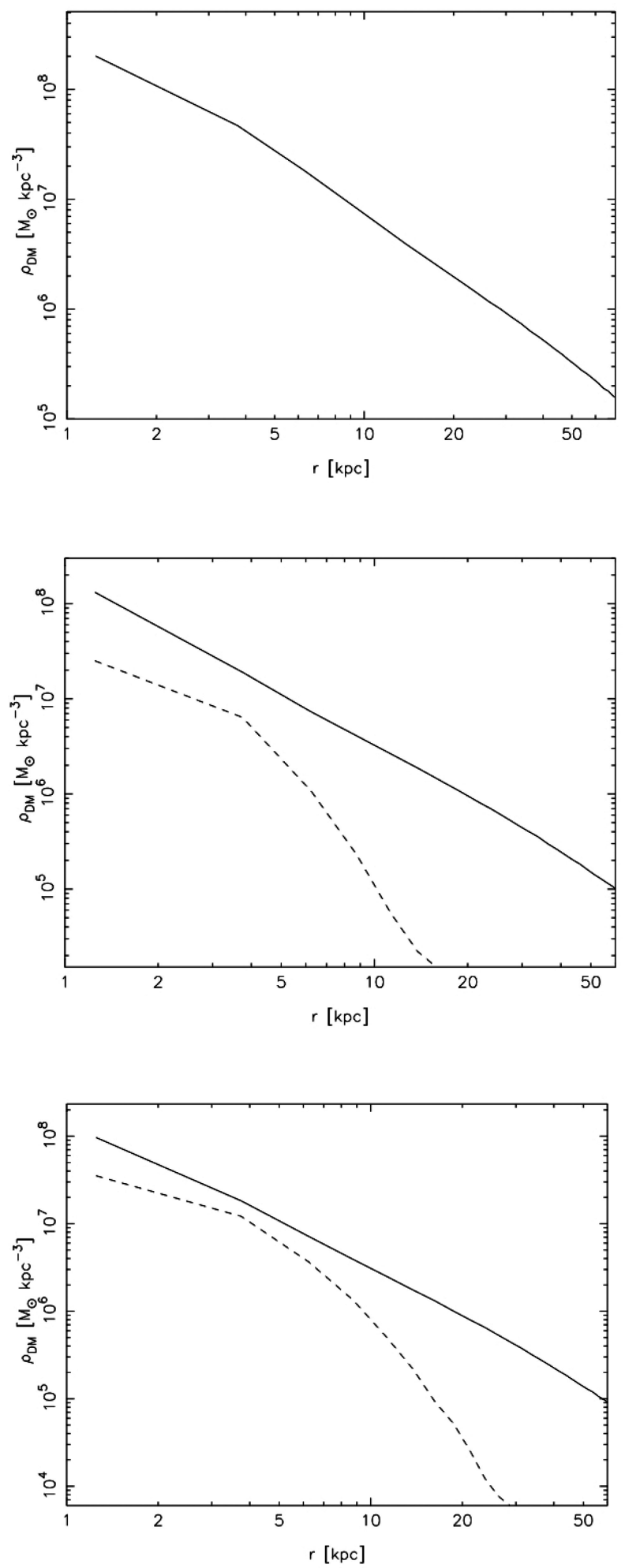

dashed line is at $z=0$. There is no profile for G01 at $z=0$ since the halo is that of the cluster. For the rest of the galaxies, refer to the online figures. Fig. A2 shows the surface brightness profile in the $V$ band for the whole galaxy (black solid), the disc stars (blue dotted) and the bulge stars (red dashed) when the galaxy is first inserted into the simulation. For the rest of the galaxies, refer to
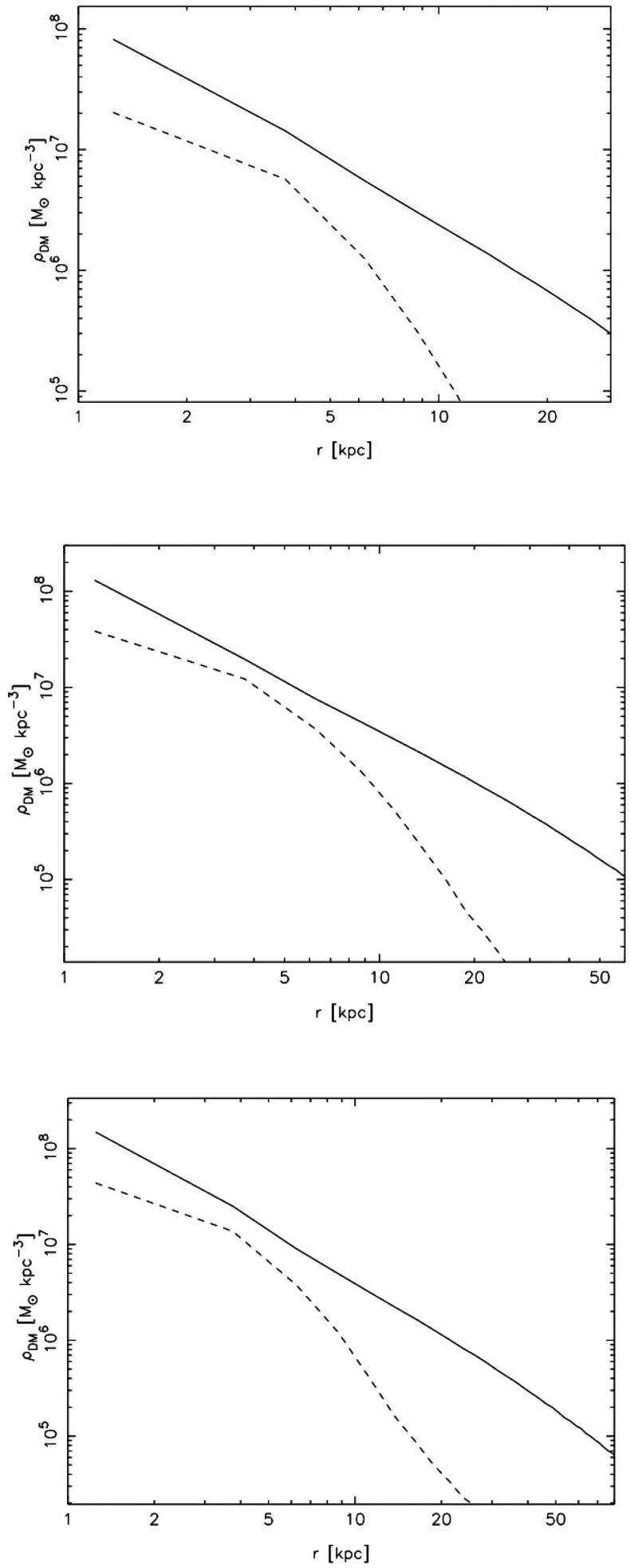

Figure A1. Dark matter volume density profile for G01-G06. The solid line is the halo at the time of insertion, and the dashed line is at $z=0$. No $z=0$ dark matter profile is shown for G01 because the galaxy is now at the centre of the cluster. The figures for the other galaxies can be found online. 

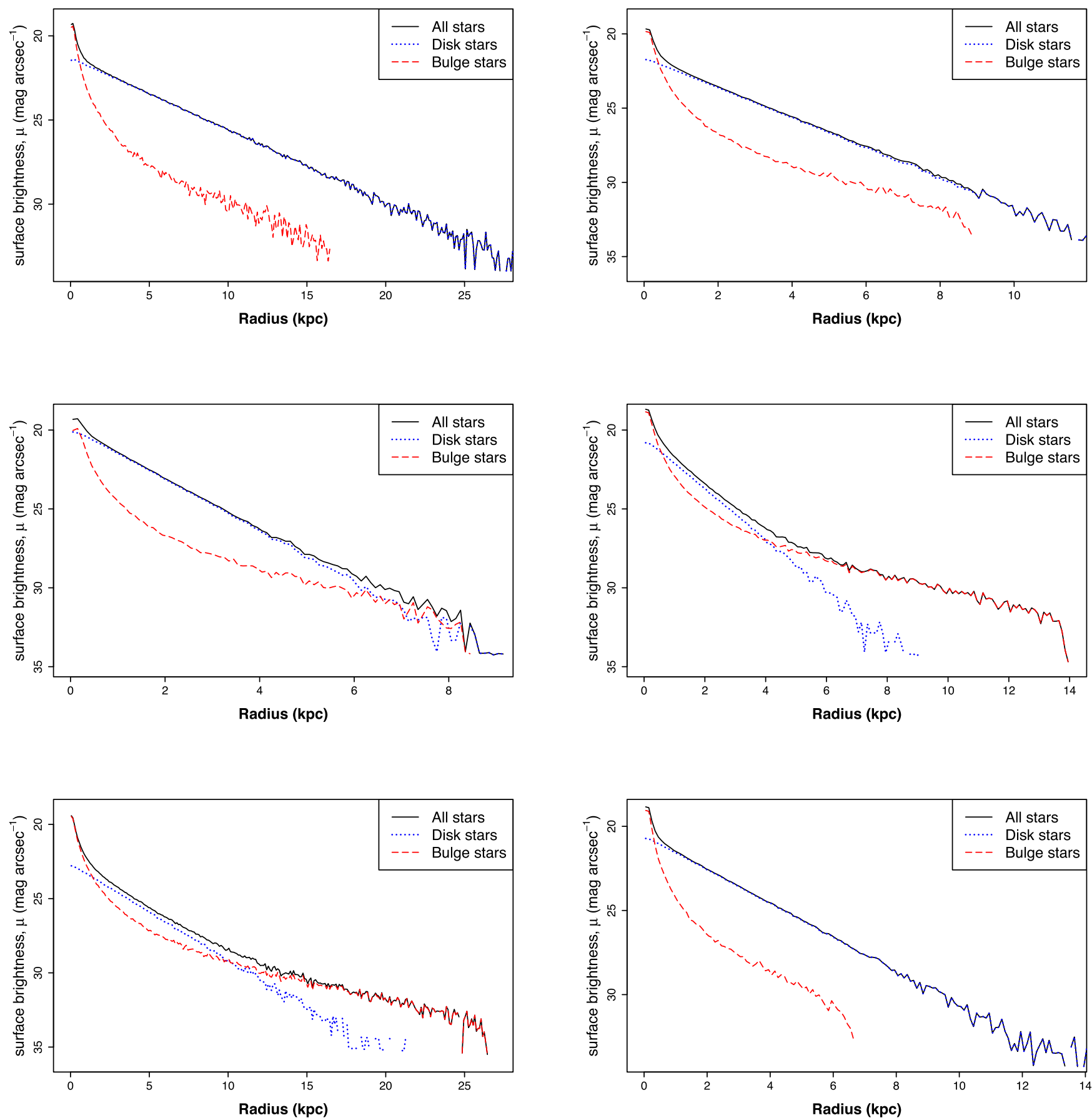

Figure A2. Surface brightness profile in the $V$ band for the whole galaxy (black solid), the disc stars (blue dotted) and the bulge stars (red dashed) when the galaxy is first inserted into the simulation for G01-G06. The figures for the other galaxies can be found online.

the online figures. Fig. A3 shows the orbit taken by that galaxy, with the galaxies moving from red to blue as time progresses. The initial insertion point is given by a brown diamond. The path moves from red to blue ending in the violet square, which indicates the final position at $z=0$. The dotted circle shows the cluster virial radius while the smaller solid circle in the centre shows the halflight radius of the $\mathrm{cD}$ galaxy at $z=0$. For the rest of the galaxies, refer to the online figures. 

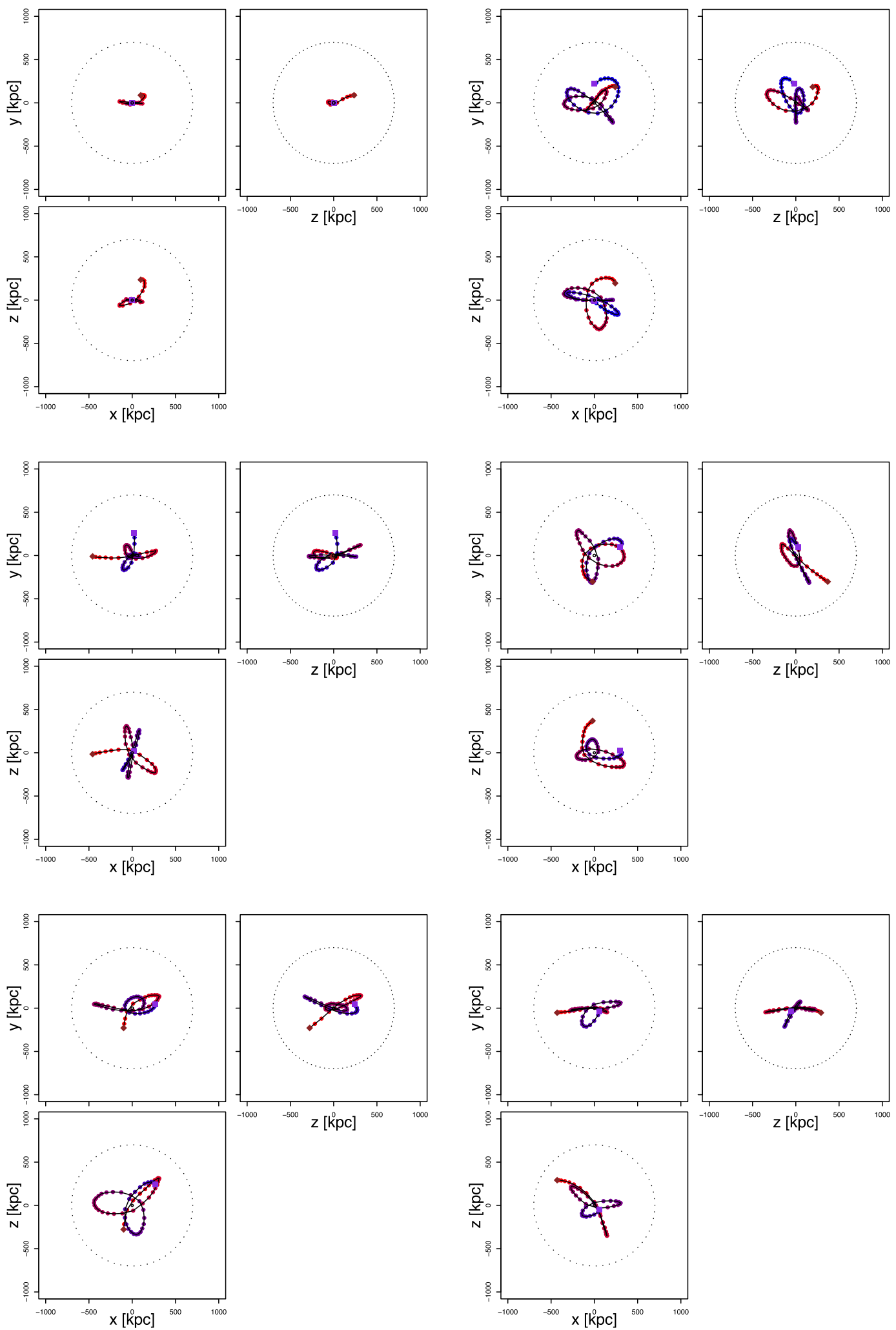

Figure A3. The orbit taken by galaxies G01-G06. The initial insertion point is given by a brown diamond. The path moves from red to blue ending in the violet square, which indicates the final position at $z=0$. The dotted black circle shows the virial radius, and the central blue circle shows the half-mass radius of model G01, both at $z=0$. The figures for the other galaxies can be found online.

This paper has been typeset from a $\mathrm{T}_{\mathrm{E}} \mathrm{X} / \mathrm{L} \mathrm{T}_{\mathrm{E}} \mathrm{X}$ file prepared by the author. 